\title{
The Effect of Ongoing Vitamin D and Low-Fat Milk Intake on Bone Metabolism in Female High-School Endurance Runners
}

\author{
Aoi Ikedo ${ }^{\text {a }}$, Takuma Arimitsua ${ }^{\text {a }}$ Toshiyuki Kurihara ${ }^{a}$, Kumiko Ebia \\ Satoshi Fujita ${ }^{a, b}$
}

\begin{abstract}
Background: Vitamin D and calcium are essential nutrients for bone health. In addition, vitamin D suppresses inflammatory cytokines and increases bone resorption. Therefore, improvements in bone health by calcium and vitamin D supplementation have the potential to not only improve calcium metabolism but also suppress inflammation associated with exercise training. The purpose of this study was to determine whether ongoing vitamin D supplementation and low-fat milk intake by female high-school endurance runners would improve bone metabolism by suppressing inflammatory cytokines and the parathyroid hormone $(\mathrm{PTH})$.
\end{abstract}

Methods: Twenty female high-school runners were assigned to a vitamin D supplement and low-fat milk intake group (MKD) or a control group (CON). Participants in the MKD group consumed a vitamin D supplement (1,000 IU/day) and low-fat milk (Ca $315 \mathrm{mg} /$ day) for 6 months. Bone mineral density measurements, blood samples, and questionnaires (regarding menses and diet) were carried out. The UMIN Clinical Trials Registry number is UMIN000027854.

Results: The 25-hydroxyvitamin D $(25(\mathrm{OH}) \mathrm{D})$ concentration in MKD was sustained and PTH concentration was decreased regardless of the state of menses. The correlation coefficients of $25(\mathrm{OH}) \mathrm{D}$ or PTH concentrations and bone metabolism markers were analyzed by partial correlation coefficient via adjusting the model for frequency of menses. CTX and 25(OH)D concentration were significantly and inversely correlated at baseline $(\mathrm{r}=-0.61, \mathrm{P}<0.01), 3$ months $(\mathrm{r}=$ $-0.54, \mathrm{P}=0.02)$, and 6 months $(\mathrm{r}=-0.53, \mathrm{P}=0.02)$. CTX and PTH were significantly and positively correlated at 3 months $(\mathrm{r}=0.63, \mathrm{P}$ $<0.01)$ and 6 months $(\mathrm{r}=0.52, \mathrm{P}=0.02)$. The bone alkaline phosphatase (BAP)/CTX ratio and $25(\mathrm{OH}) \mathrm{D}$ concentration were significantly and positively correlated at 3 months $(\mathrm{r}=0.59, \mathrm{P}=0.01)$ and 6 months $(\mathrm{r}=0.56, \mathrm{P}=0.01)$. The $\mathrm{BAP} / \mathrm{CTX}$ ratio and $\mathrm{PTH}$ were

Manuscript submitted September 22, 2017, accepted October 9, 2017

aFaculty of Sport and Health Science, Ritsumeikan University, Kusatsu, Shiga, Japan

${ }^{\mathrm{b} C}$ Corresponding Author: Satoshi Fujita, Faculty of Sport and Health Science, Ritsumeikan University, 1-1-1, Nojihigashi, Kusatsu, Shiga 525-8577, Japan. Email:safujita@fc.ritsumei.ac.jp

doi: https://doi.org/10.14740/jocmr3209w significantly and inversely correlated at 3 months $(\mathrm{r}=-0.59, \mathrm{P}=0.01)$ and 6 months $(\mathrm{r}=-0.58, \mathrm{P}<0.01)$.

Conclusions: This study suggested that vitamin D and low-fat milk supplementation improves bone metabolism by sustaining the $25(\mathrm{OH})$ D concentration and decreasing the PTH concentration in female highschool endurance runners regardless of the state of menses.

Keywords: Adolescent runners; Bone metabolism; Parathyroid hormone; 25-hydroxyvitamin D; Inflammatory cytokines

\section{Introduction}

Physical activity is associated with an increase in bone mineral accumulation during childhood and with a decrease in the bone loss rate after peak bone mass is reached [1]. However, some athletes, such as endurance runners, have been observed to have low bone mineral density (BMD) and subsequent stress fractures [2-4]. These result from the excess bone resorption caused by an increase in exercise volume and the abnormal repetitive load on the bones [5]. In addition, low BMD in female athletes has been reported caused by amenorrhea for low energy availability [2-4].

The dietary strategy for prevention of low BMD and stress fractures in athletes is calcium and vitamin D supplementation. In a 2-year cohort study of female endurance runners, a high intake of dairy products (including calcium, vitamin D, and protein) was observed to increase the BMD and decrease the incidence of stress fractures [6]. In addition, calcium and vitamin D supplementation during military training was observed to be associated with increased ionized calcium levels, maintenance of parathyroid hormone (PTH) concentration, a decreased bone resorption rate, and improved BMD and bone structure [7]. Thus, the benefits of calcium and vitamin D supplementation have been reported in many previous studies [6-8]. Vitamin D is thought to aid in calcium absorption from the small intestine, thus increasing the serum calcium concentration and decreasing the PTH concentration, thus decreasing bone resorption $[9,10]$.

On the other hand, inflammatory cytokines were reported to accelerate bone resorption and suppress bone formation [11]. In a previous study, tumor necrosis factor-alpha (TNF- $\alpha$ ), an inflammatory cytokine, was increased after high-intensity 
exercise, and the percent change of TNF- $\alpha$ showed positive correlations with the percent change of bone resorption markers [12]. On the contrary, vitamin D has been shown to suppress inflammatory cytokines such as interleukin (IL)-6, IL8 , and TNF- $\alpha$ via vitamin D receptors [13]. Furthermore, the 25-hydroxyvitamin D (25(OH)D) and TNF- $\alpha$ concentrations in runners reportedly have a reverse correlation [14]. Therefore, improvement in bone health by calcium and vitamin D supplementation has the potential to not only improve calcium metabolism but also to suppress inflammation.

The purpose of this study was to determine whether the intake of vitamin D supplements and low-fat milk for 6 months in female high-school endurance runners would improve bone metabolism through suppression of PTH and inflammatory cytokines.

\section{Materials and Methods}

\section{Subjects}

This study recruited 29 high-school female endurance runners (age $16.4 \pm 0.6$ years). The trial period was 6 months from September 2016 to April 2017. During the study, the subjects adhered to the following guidelines: 1) maintain a normal lifestyle, 2) take vitamin D supplements and low-fat milk as specified every day for those in the study group, 3) refrain from the use of supplements, health foods, medicines (including general medicines), or quasi-drugs that may affect this research, and 4) report to the research director when consulting medical institutions or using medicine. During the trial period, nine people were excluded (reasons included sickness, inadequate supplement or milk intake, and discontinued training), and finally 20 subjects were analyzed (Fig. 1).

\section{Study design}

This was a 6-month randomized controlled trial. Subjects were assigned to 1) the vitamin D supplement + low-fat milk (MKD) group $(\mathrm{n}=10$, age $16.3 \pm 0.5$ years) or 2$)$ the control $(\mathrm{CON})$ group $(\mathrm{n}=10$, age $16.3 \pm 0.6$ years $)$ that received no intervention. Before and after the 6-month trial period, we measured the participants' BMD, fat mass, and fat-free mass (FFM) using dual-energy X-ray absorptiometry (DXA). In addition, at baseline, after 3 months, and after 6 months, we performed blood sampling and administered questionnaires (assessment of menses and running distance). The study protocol was approved by the Ethics Committee for Human Experiments at Ritsumeikan University and was conducted in accordance with the Declaration of Helsinki. All subjects and legal guardians of subjects provided informed consent for participation in this study.

\section{Nutrient supplementation}

The study group participants consumed 1,000 IU of vitamin D
(Nature Made, Super D, Otsuka Pharmaceutical Co., Ltd, Japan; $0.96 \mathrm{kcal}$ energy, $0-0.1 \mathrm{~g}$ protein, $0-0.1 \mathrm{~g}$ fat, $0.231 \mathrm{~g}$ carbohydrates, 1,000 IU vitamin D, and 0 - $2 \mathrm{mg}$ sodium/pill) and $200 \mathrm{~mL}$ of low-fat milk (SAVAS MILK PROTEIN, Meiji Co., Ltd; $107 \mathrm{kcal}$ energy, $10.0 \mathrm{~g}$ protein, $3.2 \mathrm{~g}$ fat, $9.6 \mathrm{~g}$ carbohydrates, $0.22 \mathrm{~g}$ table salt equivalent, $315 \mathrm{mg}$ calcium, and 0.65 $\mathrm{mg}$ vitamin $\left.\mathrm{B}_{6}\right)$. Vitamin D supplementation was taken 1 pill a day after dinner. The low-fat milk $(200 \mathrm{~mL})$ was consumed at the club on the practice day prior to exercise (up to $1 \mathrm{~h}$ before), and on days off, the milk was consumed during free time.

\section{Assessment of menses and diet}

The status of menses (age of menarche, frequency of menses (times/year), and menstrual cycle) was assessed using a questionnaire. In a 3-day diet record using a food weighing method, daily energy and nutrient intake were assessed at baseline.

\section{Blood analysis}

Blood samples were collected from a vein in the cubital fossa at rest in a fasting state (8:00 - 10:00 am). Blood samples were centrifuged at $3,000 \mathrm{rpm}$ at $4{ }^{\circ} \mathrm{C}$ for $10 \mathrm{~min}$, and separated serum samples were frozen and stored at $-20{ }^{\circ} \mathrm{C}$ until analysis. The levels of 25(OH)D (25-OH Vitamin D Xpress ELISA Kit), TNF- $\alpha$ (Quantikine ${ }^{\circledR}$ ELISA, R\&D systems), and type I collagen cross-linked C-telopeptides (CTX; FRELISA ${ }^{\circledR} \beta$ CrossLaps $^{\circledR}-\mathrm{N}$, Fujirebio Inc.) were measured using an enzyme-linked immunoassay (ELISA). The intra- and inter-assay $\mathrm{CV}$ values were $4.9-9.4 \%$ and $7.4-8.8 \%$ for $25(\mathrm{OH}) \mathrm{D}, 3.1$ $8.7 \%$ and $7.2-10.4 \%$ for TNF- $\alpha$, and $4.6-6.1 \%$ and $2.1-5.3 \%$ for CTX, respectively. PTH and bone alkaline phosphatase (BAP) levels were measured using an electrochemiluminescence immunoassay (ECLIA) (Medic, Inc., Japan). Intra- and inter-assay $\mathrm{CV}$ values were within $15 \%$.

\section{Body composition and BMD}

The body mass, fat mass, percent body fat, appendicular FFM, and bone mass were evaluated by DXA (Lunar Prodigy; GE Healthcare, Tokyo, Japan). During DXA measurements, subjects remained in a supine position. To evaluate the whole body scans, we used enCORE version 15 software (GE Medical Systems Lunar, Tokyo, Japan), which automated measurements of FFM and fat-mass (arms, legs, torso, gynoid (gluteal area), and total body), BMD (total bone less head (TBLH), arms, spine, pelvis, and legs), and percent body fat. For screening of athletes younger than 20 years of age who are at risk for low BMD, measurement of TBLH BMD is recommended [15].

\section{Statistical analysis}

Data are shown as the mean \pm standard deviation (SD) un- 


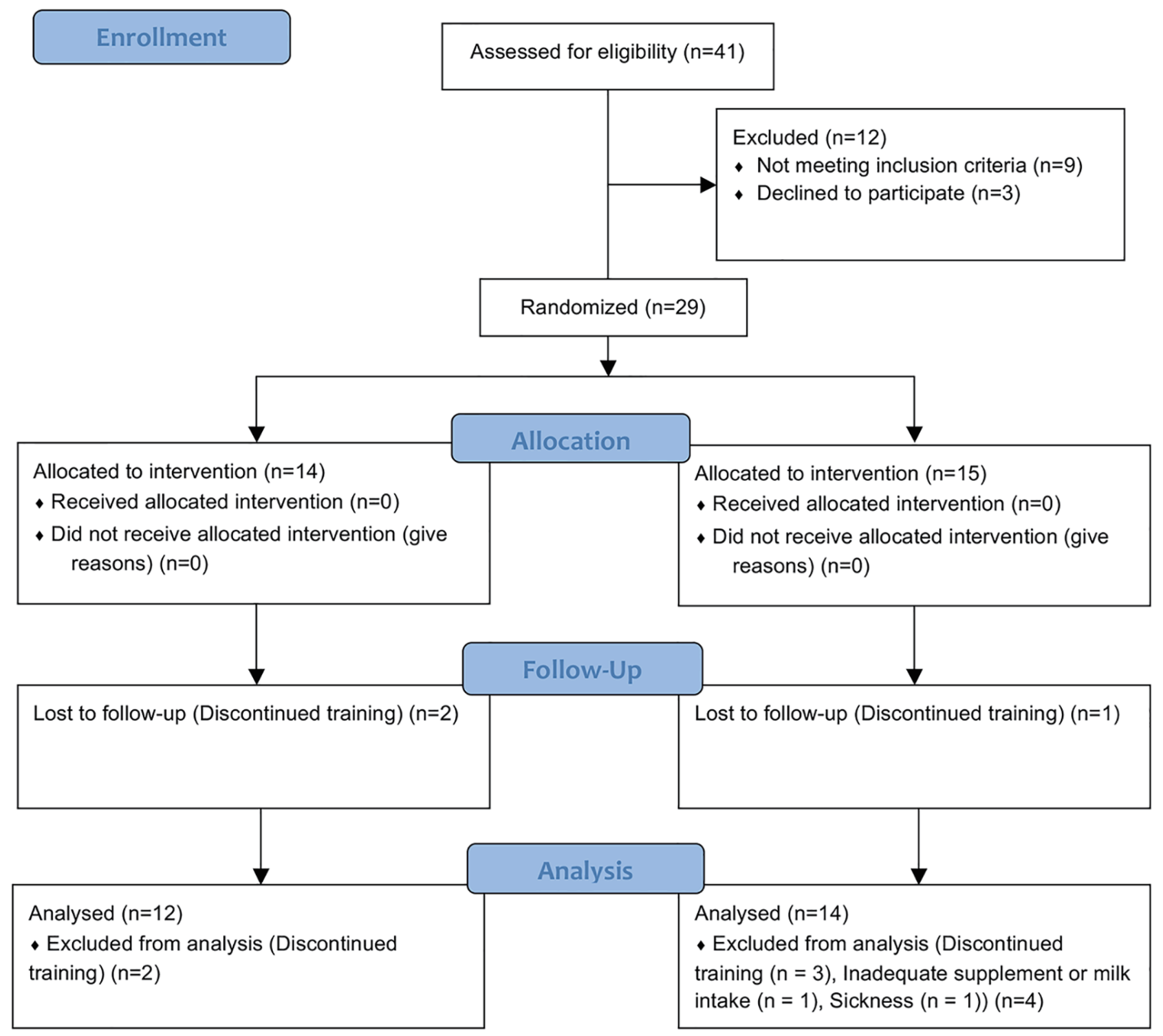

Figure 1. Study flow diagram.

less otherwise noted. The concentration of bone formation markers (BAP) divided by the concentration of resorption markers (CTX) was used to calculate the bone formation to resorption ratios (BAP/CTX). The calculated ratios were used to assess the change of bone turnover balance [16]. Comparison of baseline values between groups was evaluated using unpaired $t$-tests. Differences in time (baseline, 3 months, and 6 months) and group (MKD and CON) were evaluated with repeated two-way ANOVAs for subject characteristics, BMD, and biomarkers. The Bonferroni method for post hoc analysis was performed when time $\times$ group interactions were observed. To determine the effects of supplementation on 25(OH)D concentrations, PTH, TNF- $\alpha$, and bone metabolism markers, we first calculated the changes from baseline by subtracting the baseline values from the 3-month and 6-month trial values. Then we applied an anal- ysis of covariance. To identify whether the significant differences were influenced by the baseline values of $25(\mathrm{OH}) \mathrm{D}$ and PTH concentration, TNF- $\alpha$, and bone metabolism markers, we adjusted for the baseline values of each biomarker. We also controlled for running distance and frequency of menses, because these were observed to be significantly different between the groups $(\mathrm{P}=0.03)$. Correlations between indexes were evaluated using the Pearson correlation coefficient. The correlation coefficients between $25(\mathrm{OH}) \mathrm{D}$ or PTH concentrations and bone metabolism markers were analyzed by partial correlation coefficients with the model adjusted for the frequency of menses. The statistical significance level was set at 5\%, and a level below $10 \%$ was defined as a tendency. Statistical analysis was performed using SPSS Statistics Version 24.0 for Microsoft Windows (IBM Corp., Tokyo, Japan). The UMIN Clinical Trials Registry number 
Table 1. Subject Characteristics

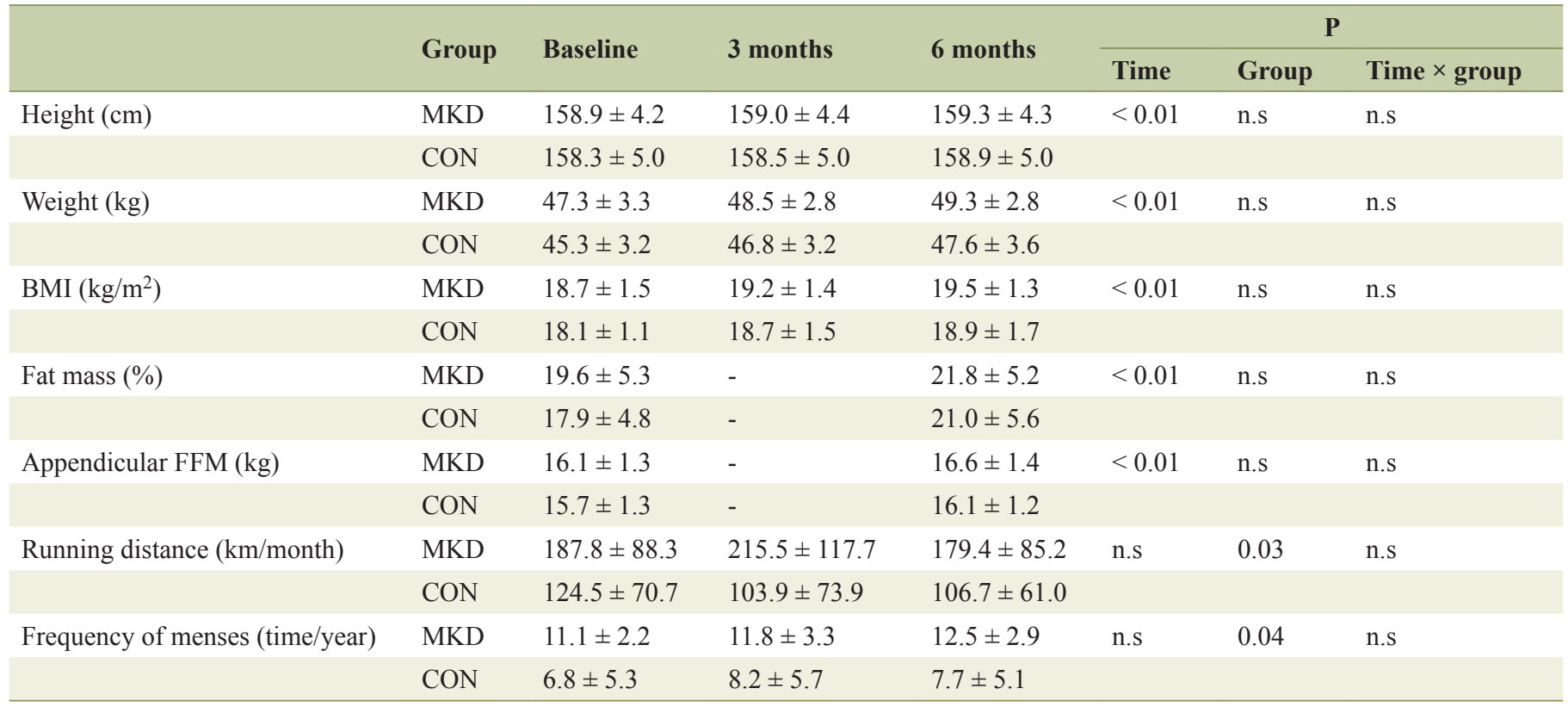

Values are represented as mean $\pm S D$. Differences in time (baseline, 3 months, and 6 months) and group (MKD and CON) were evaluated with repeated two-way ANOVAs for subject characteristics. Because fat mass and fat-free mass (FFM) were measured in before and after the 6-month trial period only, the values in 3 months were not measured (-). MKD: vitamin D supplement + low-fat milk group; CON: control group.

is UMIN000027854.

\section{Results}

\section{Subject characteristics}

Subject characteristics are shown in Table 1. There were no significant differences in any of subject characteristics between groups at baseline. None of the confounding factors were significant for time $\times$ group interactions. Running distance $(P=0.03)$ and frequency of menses $(P=0.04)$ were significantly different between the groups (Table 1). Age of menarche was not significantly different between the groups $(\mathrm{P}=0.69)(\mathrm{CON}: 13.2 \pm 1.0$ years, two subjects were delayed menarche; MKD: $13.5 \pm 1.4$ years; two subjects in CON group who reported menarche delay were excluded from the calculation). In addition, energy and nutrient intakes at baseline were not significantly different between the groups (energy: MKD 2,103 $\pm 366 \mathrm{kcal} /$ day, CON 2,043 $\pm 340 \mathrm{kcal} /$ day, $\mathrm{P}=0.71$, calcium: MKD $445 \pm 265 \mathrm{mg} /$ day, CON $495 \pm 106$ $\mathrm{mg} /$ day, $\mathrm{P}=0.59$, vitamin $\mathrm{D}: \mathrm{MKD} 10.5 \pm 7.5 \mu \mathrm{g} /$ day, $\mathrm{CON}$ $16.5 \pm 18.8 \mu \mathrm{g} /$ day, $\mathrm{P}=0.37$ ).

\section{BMD}

Changes in the subjects' BMD are shown in Table 2. There were no significant differences in any of BMDs between groups at baseline. The change in BMD for all sites tested was not significant for time $\times$ group interactions.

\section{Biomarkers}

The changes in concentration of $25(\mathrm{OH}) \mathrm{D}, \mathrm{PTH}, \mathrm{TNF}-\alpha$, and bone metabolism markers are shown in Table 3 . There were no significant differences in baseline values of $25(\mathrm{OH}) \mathrm{D}$, PTH, or bone metabolism markers between groups. TNF- $\alpha$ concentration at baseline was significantly higher in the MKD group compared with that in the CON group $(\mathrm{P}=0.02)$. A significant effect of time $(\mathrm{P}<0.01)$ and time $\times$ group interaction $(\mathrm{P}<0.01)$ was observed in $25(\mathrm{OH}) \mathrm{D}$ concentration. In a post hoc analysis, $25(\mathrm{OH}) \mathrm{D}$ concentration in the CON group significantly decreased from baseline to 3 months $(\mathrm{P}<$ $0.01)$ and from baseline to 6 months $(\mathrm{P}<0.01)$. In contrast, the $25(\mathrm{OH}) \mathrm{D}$ concentration in the MKD group did not change significantly during the trial period. Time $\times$ group interaction $(P=0.04)$ exhibited a significant effect on TNF- $\alpha$ concentration. TNF- $\alpha$ concentration at baseline was significantly higher in the MKD group $(1.08 \pm 0.28 \mathrm{pg} / \mathrm{mL})$ compared with that in the CON group $(0.71 \pm 0.38 \mathrm{pg} / \mathrm{mL})(\mathrm{P}=0.02)$. Moreover, TNF- $\alpha$ concentration in the MKD group decreased significantly from baseline to 6 months $(\mathrm{P}=0.02)$. PTH concentration was significant effect of time $(\mathrm{P}<0.01)$, and tend effect of time $\times$ group interaction $(\mathrm{P}=0.06)$. However, the concentration of the bone metabolism markers did not change significantly during the trial period.

\section{Association between vitamin D supplement and low-fat milk intake and biomarkers}

The association between biomarkers and vitamin D supple- 
Table 2. Changes in BMD

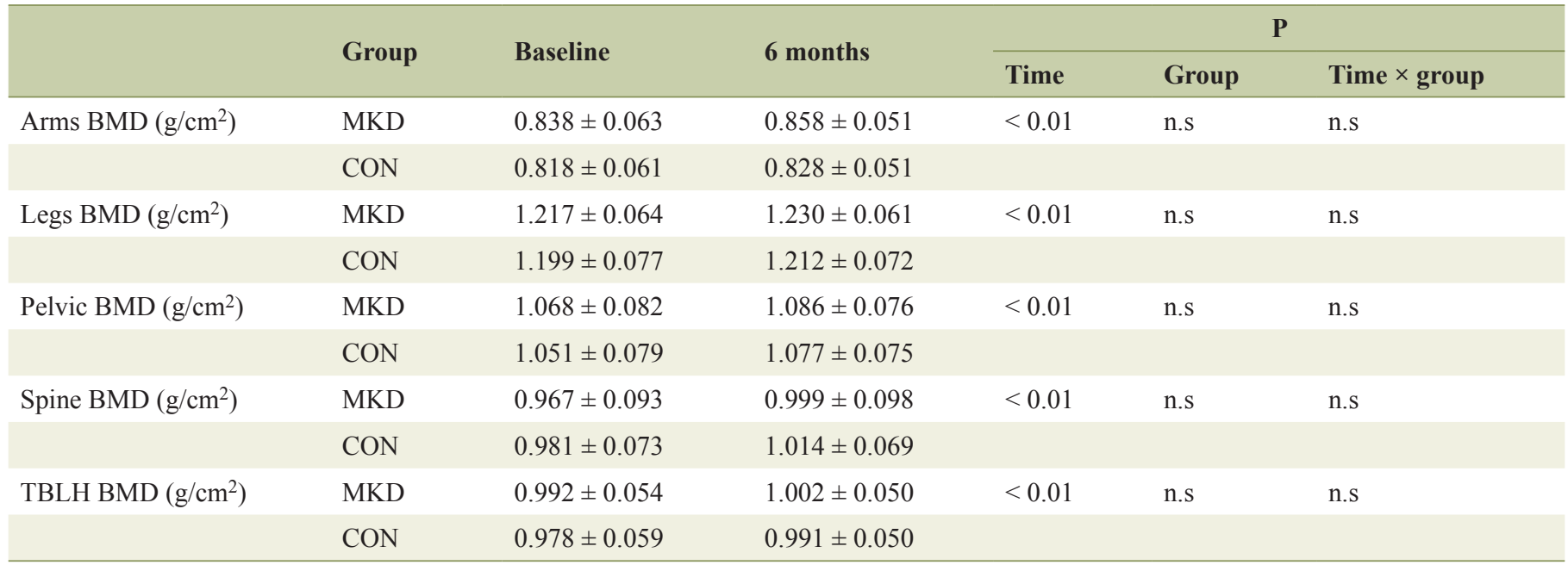

Values are represented as mean \pm SD. Differences in time (baseline, 3 months, and 6 months) and group (MKD and CON) were evaluated with repeated two-way ANOVAs for BMD. TBLH: total bone less head; MKD: vitamin D supplement + low-fat milk group; CON: control group.

mentation and low-fat milk intake is shown in Table 4. The change in concentration of each biomarker in both groups was compared after each biomarker was adjusted for baseline values, frequency of menses, and running distance. The decrease in $25(\mathrm{OH}) \mathrm{D}$ concentration from baseline to 3 months in the MKD group was significantly attenuated compared to that in the CON group even after adjusting for baseline values and frequency of menses (model 1, 2, 3: $\mathrm{P}<0.01$ ). Although the decrease in $25(\mathrm{OH}) \mathrm{D}$ concentration from baseline to 6 months in the MKD group was significantly attenuated compared to that in the CON group, even after adjusting for baseline values and frequency of menses (model 1: $\mathrm{P}<$
0.01 ; model 2: $\mathrm{P}=0.02)$, the change was not significant at 6 months after adjusting for baseline values, frequency of menses, and running distance (model $3, \mathrm{P}=0.11$ ). PTH concentration in the MKD group decreased significantly from baseline to 3 months compared with that in the CON group even after adjusting for baseline values, frequency of menses, and running distance (model 1: $\mathrm{P}=0.03$; model 2: $\mathrm{P}=0.01$; model 3: $\mathrm{P}=0.04)$. However, the change in PTH concentration from baseline to 6 months was not significantly different between the groups (model 1: $\mathrm{P}=0.10$; model 2: $\mathrm{P}=0.18$; model 3: $\mathrm{P}=0.77)$. TNF- $\alpha, \mathrm{CTX}$, and BAP concentrations were not significantly different between the groups during

Table 3. Changes in 25(OH)D, PTH, TNF- $\alpha$, and Bone Metabolism Marker Concentrations

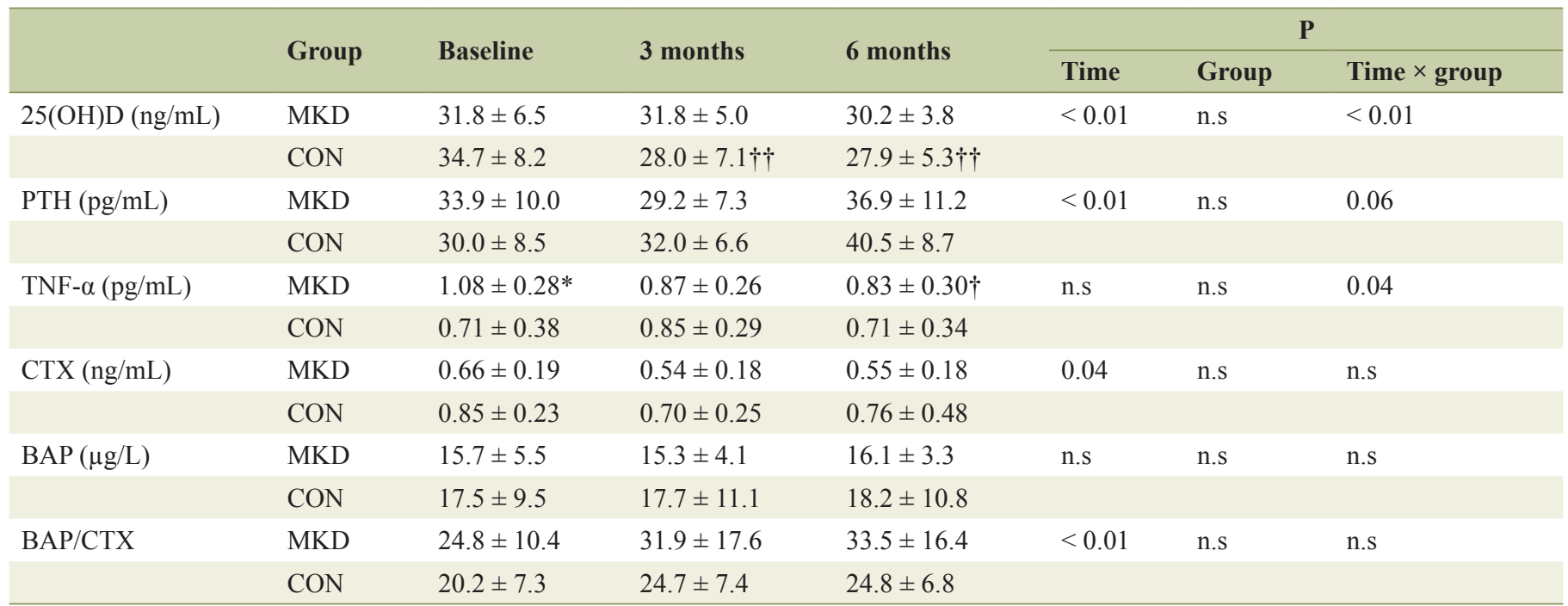

Values are represented as mean \pm SD. Differences in time (baseline, 3 months, and 6 months) and group (MKD and CON) were evaluated with repeated two-way ANOVAs for biomarkers. ${ }^{*} \mathrm{P}<0.05 \mathrm{vs}$. CON, $\dagger \mathrm{P}<0.05, \dagger \dagger \mathrm{P}<0.01 \mathrm{vs}$. Baseline. BAP/CTX: bone formation to resorption ratio; MKD: vitamin D supplement + low-fat milk group; CON: control group. 
Table 4. Effect of Vitamin D Supplementation and Low-Fat Milk Intake on Each Biomarker

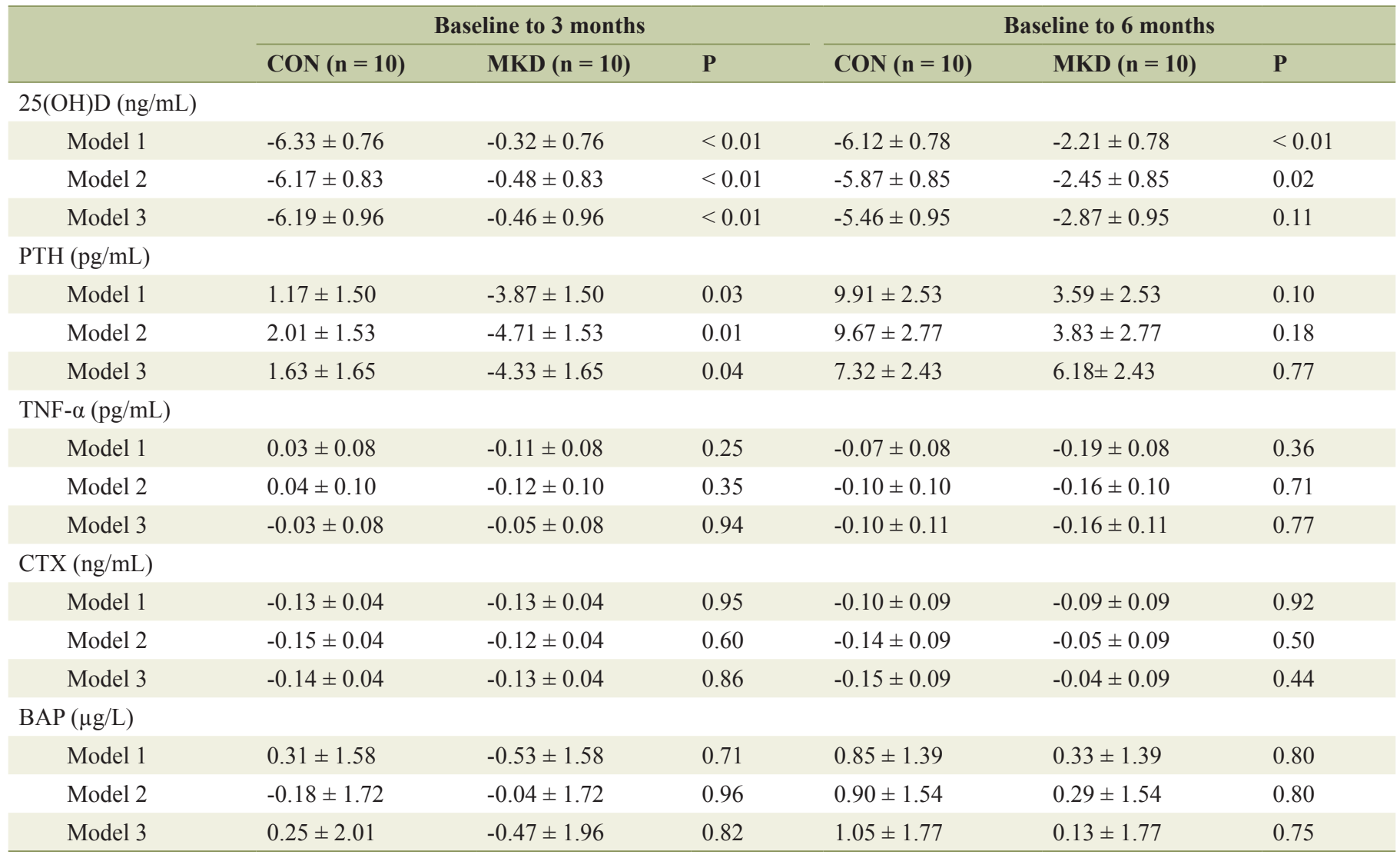

Values are represented as mean \pm SE. The change in concentration of each biomarker in both groups was compared after each biomarker was adjusted for Baseline values, frequency of menses, and running distance, respectively. Model 1: baseline value. Model 2: baseline value, frequency of menses (baseline value). Model 3: baseline value, frequency of menses (baseline value), running distance (baseline value). MKD: vitamin D supplement + low-fat milk group; CON: control group.

the trial period.

\section{Correlation between 25(OH)D or PTH concentration and bone metabolism markers}

The correlation between 25(OH)D or PTH concentration and bone metabolism markers was examined. Because CTX or BAP concentration and the frequency of menses were significantly correlated, the correlation coefficients of $25(\mathrm{OH}) \mathrm{D}$ or PTH concentrations and bone metabolism markers were analyzed by partial correlation coefficient via adjusting the model for frequency of menses. The correlation between $25(\mathrm{OH}) \mathrm{D}$ or PTH concentrations and bone metabolism markers with adjustment for frequency of menses is shown in Table 5. CTX and $25(\mathrm{OH}) \mathrm{D}$ concentration were significantly and inversely correlated at baseline, 3 months, and 6 months. CTX and PTH were significantly and positively correlated at 3 and 6 months. However, BAP was not correlated with $25(\mathrm{OH}) \mathrm{D}$ and PTH concentrations. The BAP/CTX ratio and $25(\mathrm{OH}) \mathrm{D}$ concentration were significantly and positively correlated at 3 and 6 months. The BAP/CTX ratio and PTH were significantly and inversely correlated at 3 and 6 months. In addition, 25(OH)D and PTH concentrations were significantly and inversely correlated at baseline $(\mathrm{r}=-0.50, \mathrm{P}=0.03)$ and 24 weeks $(\mathrm{r}=-0.64$, $\mathrm{P}<0.01)$. PTH and $25(\mathrm{OH}) \mathrm{D}$ concentrations at 3 months were inversely correlated $(\mathrm{r}=-0.44, \mathrm{P}=0.051)$.

\section{Discussion}

The purpose of this randomized controlled trial was to determine whether vitamin D supplementation and low-fat milk intake for 6 months by female high-school endurance runners resulted in improved bone metabolism by suppressing the concentration of PTH and inflammatory cytokines. The main finding of our study was that the $25(\mathrm{OH}) \mathrm{D}$ concentration was sustained and PTH concentration was decreased by vitamin $\mathrm{D}$ and low-fat milk supplementation regardless of the state of menses. In comparison to bone metabolism markers with $25(\mathrm{OH}) \mathrm{D}$ or PTH concentration, we found that high $25(\mathrm{OH})$ $\mathrm{D}$ and low PTH concentration decreased CTX and increased the BAP/CTX ratio (increased bone formation ratio) regardless of the state of menses. In addition, 25(OH)D and PTH concentrations were significantly and inversely correlated. Accordingly, vitamin D and low-fat milk supplementation 
Table 5. The Correlation Between 25(OH)D or PTH Concentrations and Bone Metabolism Markers With Adjustment for Frequency of Menses

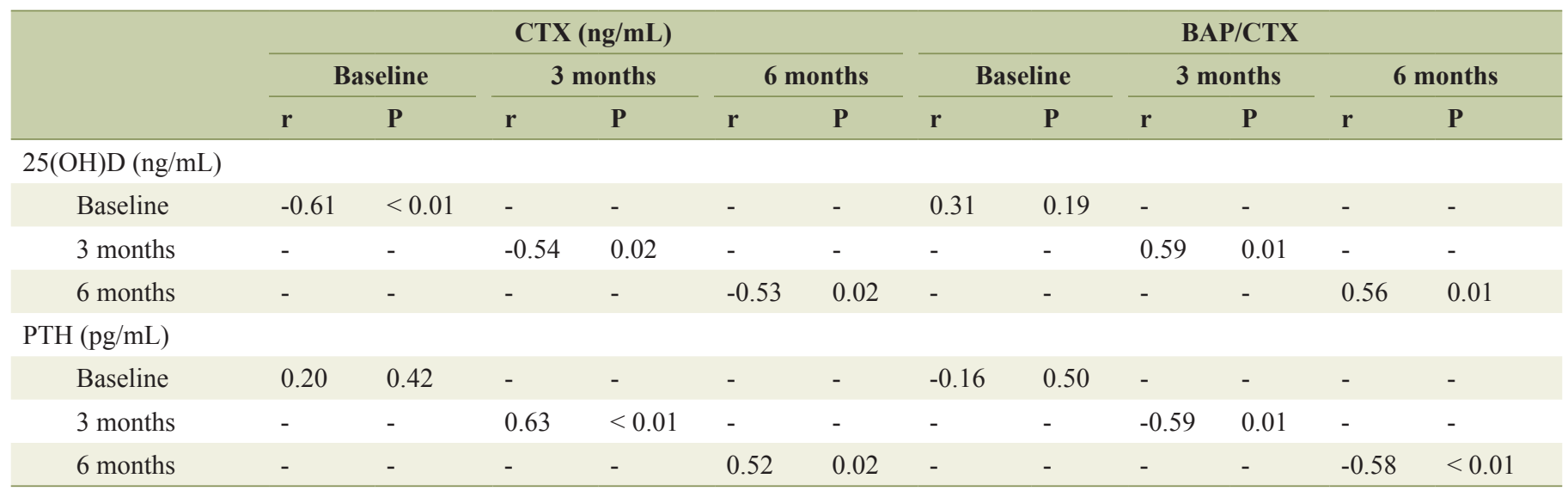

Pooled analysis by partial correlation coefficient. Values are $r$ and $P$ values.

improved bone metabolism while sustaining 25(OH)D concentration and decreasing PTH concentration regardless of the state of menses.

In $25(\mathrm{OH}) \mathrm{D}$ concentration, after adjusting for the frequency of menses and running distance, no significant change was observed at 6 months. Thus, the $25(\mathrm{OH}) \mathrm{D}$ concentration may have been influenced by the running distance. The decrease in $25(\mathrm{OH}) \mathrm{D}$ concentration has previously been reported during basic military training in male and female soldiers [17-19]. Although the mechanism is still unclear, an increased running distance may potentially influence increased PTH concentration. PTH concentration has been reported to be transient-increase dependent on exercise intensity and duration [20, 21]. In addition, teriparatide (recombinant human PTH), a therapeutic osteoporosis drug, has been reported to decrease $25(\mathrm{OH})$ D concentration [22]. 25(OH)D concentration decreased during teriparatide therapy, suggesting the conversion of $25(\mathrm{OH})$ $\mathrm{D}$ to $1,25(\mathrm{OH})_{2} \mathrm{D}$ (active vitamin $\mathrm{D}$ ) metabolite. From these previous studies, an increased running distance can potentially decrease the $25(\mathrm{OH}) \mathrm{D}$ concentration by accelerating the conversion of $25(\mathrm{OH}) \mathrm{D}$ to $1,25(\mathrm{OH})_{2} \mathrm{D}$ by increasing the PTH concentration during exercise. However, because this study could not determine the underlying relationships, future study is needed.

Vitamin D and low-fat milk supplementation significantly decreased the PTH concentration from baseline to 3 months even after adjusting for the confounders. However, PTH concentration did not change significantly from baseline to 6 months, suggesting that the changes were influenced by the changes in $25(\mathrm{OH}) \mathrm{D}$ concentration. Although the change in $25(\mathrm{OH}) \mathrm{D}$ concentrations in the CON group did not differ between baseline and 3 months (approximately $-6.3 \mathrm{ng} / \mathrm{mL}$ ) and between baseline and 6 months (approximately $-6.1 \mathrm{ng} / \mathrm{mL}$ ), in the MKD group, the concentration decreased between baseline and 6 months (approximately $-2 \mathrm{ng} / \mathrm{mL}$ ) compared with that between baseline and 3 months (approximately $-0.3 \mathrm{ng} / \mathrm{mL}$ ). The $25(\mathrm{OH}) \mathrm{D}$ concentration likely decreases more during these seasons (winter season) owing to fewer sunshine hours [17]. The 3 months measurement was performed in December or January, and the 6 months measurement was performed in March or April. Previous study of Japanese indoor and outdoor female athletes reported that the $25(\mathrm{OH}) \mathrm{D}$ concentration was lowest in March and highest in September, and December was significantly lower compared with September [23]. The change in $25(\mathrm{OH}) \mathrm{D}$ concentration of this study was akin to previous study. In addition, a correlation was observed between $25(\mathrm{OH})$ $\mathrm{D}$ and PTH concentrations. Therefore, the seasonal variations in $25(\mathrm{OH}) \mathrm{D}$ concentration suggested that was influenced to the change in PTH concentration.

In this study, regardless of the frequency of menses, vitamin D and low-fat milk supplementation was associated with a sustained 25(OH)D concentration and a decreased PTH concentration, and a significant correlation was observed between bone metabolism markers and $25(\mathrm{OH}) \mathrm{D}$ or PTH concentration. In a previous study, when the effects of calcium and vitamin D supplementation were studied in female navy recruits, the stress fracture incidence rate in female recruits with amenorrhea decreased from $91 \%$ to $83 \%$ [8]. Thus, although the state of menses negatively affects bone metabolism, calcium and vitamin D supplementation can potentially attenuate the negative effect of menstrual irregularity.

On the contrary, vitamin D and low-fat milk supplementation did not suppress TNF- $\alpha$, and TNF- $\alpha$ was not associated with bone metabolism. This may be because the TNF- $\alpha$ concentration of the subjects was low at baseline. The TNF- $\alpha$ concentration $(1.1 \pm 0.3 \mathrm{pg} / \mathrm{mL})$ of the present study subjects was low levels and was not comparable to that of patients with type 2 diabetes $(13 \pm 8.6 \mathrm{pg} / \mathrm{mL})$, in whom the inflammatory suppress effects of vitamin $\mathrm{D}$ and calcium supplementation have been observed [24]. Furthermore, the decrease in the cortical bone cross-sectional area during soldiers' army training involves IL-6 polymorphism [25]. Moreover, it has been reported that vitamin D sufficiency is associated with an increase in anti-inflammatory (IL-10 and IL-13) cytokines after intense exercise [26]. Therefore, investigation of the effects of vitamin D and low-fat milk intake on other inflammatory and antiinflammatory cytokines and the association of other cytokines and bone metabolism markers is needed. 


\section{Limitation}

The present study has several limitations. This study included a relatively small sample size. Furthermore, because the state of menses was assessed by a questionnaire only, we did not evaluate sex hormones (e.g., estrogen and progesterone) or reproductive maturation factors (such as Tanner breast stage). Therefore, a larger sample size and detailed analysis of the reproductive function is needed when investigating the effect of calcium and vitamin D supplementation on bone metabolism and inflammation in the future.

\section{Conclusions}

In conclusion, this study suggested that vitamin D and low-fat milk supplementation improves bone metabolism by sustaining $25(\mathrm{OH}) \mathrm{D}$ concentration and decreasing PTH concentration, regardless of the state of menses in female high-school endurance runners.

\section{Acknowledgments}

The authors wish to acknowledge the volunteers who participated in this study.

\section{Conflict of Interest}

The authors declare no conflict of interest.

\section{Grant Support}

The Japan Dairy Association (J-milk) and the Yamaha Motor Foundation for Sports provided grants to support this study.

\section{References}

1. Kohrt WM, Bloomfield SA, Little KD, Nelson ME, Yingling VR, American College of Sports M. American College of Sports Medicine Position Stand: physical activity and bone health. Med Sci Sports Exerc. 2004;36(11):1985-1996.

2. Barrack MT, Rauh MJ, Nichols JF. Prevalence of and traits associated with low BMD among female adolescent runners. Med Sci Sports Exerc. 2008;40(12):2015-2021.

3. Bilanin JE, Blanchard MS, Russek-Cohen E. Lower vertebral bone density in male long distance runners. Med Sci Sports Exerc. 1989;21(1):66-70.

4. Tenforde AS, Fredericson M, Sayres LC, Cutti P, Sainani KL. Identifying sex-specific risk factors for low bone mineral density in adolescent runners. Am J Sports Med. 2015;43(6):1494-1504.

5. Matcuk GR, Jr., Mahanty SR, Skalski MR, Patel DB, White EA, Gottsegen CJ. Stress fractures: pathophysiolo- gy, clinical presentation, imaging features, and treatment options. Emerg Radiol. 2016;23(4):365-375.

6. Nieves JW, Melsop K, Curtis M, Kelsey JL, Bachrach LK, Greendale G, Sowers MF, et al. Nutritional factors that influence change in bone density and stress fracture risk among young female cross-country runners. PM R. 2010;2(8):740-750; quiz 794.

7. Gaffney-Stomberg E, Lutz LJ, Rood JC, Cable SJ, Pasiakos SM, Young AJ, McClung JP. Calcium and vitamin D supplementation maintains parathyroid hormone and improves bone density during initial military training: a randomized, double-blind, placebo controlled trial. Bone. 2014;68:46-56.

8. Lappe J, Cullen D, Haynatzki G, Recker R, Ahlf R, Thompson K. Calcium and vitamin d supplementation decreases incidence of stress fractures in female navy recruits. J Bone Miner Res. 2008;23(5):741-749.

9. Fleet JC. The role of vitamin D in the endocrinology controlling calcium homeostasis. Mol Cell Endocrinol. 2017;453:36-45.

10. Takahashi N, Udagawa N, Suda T. Vitamin D endocrine system and osteoclasts. Bonekey Rep. 2014;3:495.

11. Takayanagi H. New developments in osteoimmunology. Nat Rev Rheumatol. 2012;8(11):684-689.

12. Mezil YA, Allison D, Kish K, Ditor D, Ward WE, Tsiani E, Klentrou P. Response of Bone Turnover Markers and Cytokines to High-Intensity Low-Impact Exercise. Med Sci Sports Exerc. 2015;47(7):1495-1502.

13. Muller K, Bendtzen K. 1,25-Dihydroxyvitamin D3 as a natural regulator of human immune functions. J Investig Dermatol Symp Proc. 1996;1(1):68-71.

14. Willis KS, Smith DT, Broughton KS, Larson-Meyer DE. Vitamin D status and biomarkers of inflammation in runners. Open Access J Sports Med. 2012;3:35-42.

15. Gordon CM, Bachrach LK, Carpenter TO, Crabtree N, El-Hajj Fuleihan G, Kutilek S, Lorenc RS, et al. Dual energy X-ray absorptiometry interpretation and reporting in children and adolescents: the 2007 ISCD Pediatric Official Positions. J Clin Densitom. 2008;11(1):43-58.

16. Jiang J, Boyle LJ, Mikus CR, Oberlin DJ, Fletcher JA, Thyfault JP, Hinton PS. The effects of improved metabolic risk factors on bone turnover markers after 12 weeks of simvastatin treatment with or without exercise. Metabolism. 2014;63(11):1398-1408.

17. Davey T, Lanham-New SA, Shaw AM, Hale B, Cobley R, Berry JL, Roch M, et al. Low serum 25-hydroxyvitamin D is associated with increased risk of stress fracture during Royal Marine recruit training. Osteoporos Int. 2016;27(1):171-179.

18. Lutz LJ, Karl JP, Rood JC, Cable SJ, Williams KW, Young AJ, McClung JP. Vitamin D status, dietary intake, and bone turnover in female Soldiers during military training: a longitudinal study. J Int Soc Sports Nutr. 2012;9(1):38.

19. Evans RK, Antczak AJ, Lester M, Yanovich R, Israeli E, Moran DS. Effects of a 4-month recruit training program on markers of bone metabolism. Med Sci Sports Exerc. 2008;40(11 Suppl):S660-670.

20. Scott JP, Sale C, Greeves JP, Casey A, Dutton J, Fraser WD. The role of exercise intensity in the bone metabolic 
response to an acute bout of weight-bearing exercise. J Appl Physiol (1985). 2011;110(2):423-432.

21. Scott JP, Sale C, Greeves JP, Casey A, Dutton J, Fraser WD. Treadmill running reduces parathyroid hormone concentrations during recovery compared with a nonexercising control group. J Clin Endocrinol Metab. 2014;99(5):1774-1782.

22. Cosman F, Dawson-Hughes B, Wan X, Krege JH. Changes in vitamin $\mathrm{D}$ metabolites during teriparatide treatment. Bone. 2012;50(6):1368-1371.

23. Maruyama-Nagao A, Sakuraba K, Suzuki Y. Seasonal variations in vitamin $\mathrm{D}$ status in indoor and outdoor female athletes. Biomed Rep. 2016;5(1):113-117.

24. Tabesh M, Azadbakht L, Faghihimani E, Tabesh M, Es- maillzadeh A. Calcium-vitamin D cosupplementation influences circulating inflammatory biomarkers and adipocytokines in vitamin D-insufficient diabetics: a randomized controlled clinical trial. J Clin Endocrinol Metab. 2014;99(12):E2485-2493.

25. Dhamrait SS, James L, Brull DJ, Myerson S, Hawe E, Pennell DJ, World M, et al. Cortical bone resorption during exercise is interleukin-6 genotype-dependent. Eur J Appl Physiol. 2003;89(1):21-25.

26. Barker T, Martins TB, Hill HR, Kjeldsberg CR, Dixon BM, Schneider ED, Henriksen VT, et al. Vitamin D sufficiency associates with an increase in anti-inflammatory cytokines after intense exercise in humans. Cytokine. 2014;65(2):134-137. 